\title{
Premixed versus sequential administration of intrathecal fentanyl as a bupivacaine adjuvant in lower limb orthopedic surgeries: a prospective randomized controlled study
}

\author{
El Kenany S., MD
}

Department of Anesthesia ,Surgical Inten-

sive Care and Pain management, Col-

lege of Medicine, Mansoura University,

Mansoura, Egypt.

\section{Introduction}

Spinal anesthesia is the most frequently used anesthetic technique that can provide fast, safe, reliable, and effective anesthesia and early postoperative analgesia in patients scheduled to elective lower limb orthopedic surgeries, but it is of limited duration. [1] Different drugs have been added to local anesthetics (LAs) to extend the duration and

\begin{abstract}
Introduction: Intrathecal fentanyl is routinely mixed with hyperbaric bupivacaine during spinal block, but this may alter the baricity of both drugs and hence affect their spread and action.

Aim: This study aimed to compare the sequential administration of intrathecal fentanyl and bupivacaine to the routine mixing of the two drugs as regard the block characteristics, the postoperative analgesia duration, and the adverse effects.

Methods: Current prospective, controlled, randomized trial was carried out on 100 adult participants subjected to lower limb orthopedic surgeries under spinal anesthesia. Subjects were allocated randomly into two equal groups (50 each). Group P: received premixed solution of hyperbaric bupivacaine (HB) $0.5 \%$ (12.5 mg) plus $25 \mu \mathrm{g}$ of fentanyl in the same syringe and group S: received $25 \mu \mathrm{g}$ of fentanyl followed by $12.5 \mathrm{mg}$ of $\mathrm{HB}$ in sequential manner. Block characteristics, time to the first rescue pethidine request, number of participants who requested for pethidine within the first 6 postoperative hours, and adverse effects were assessed.

Results: Group $S$ patients had statistically significantly faster onset of both sensory ( $4.58 \pm 1.5$ vs $5.40 \pm 1.8 \mathrm{~min}, \mathrm{p}=0.02)$ and motor block $(5.79 \pm$ $1.5 \mathrm{~min}$ versus $6.64 \pm 1.9 \mathrm{~min}, p=0.01$ ), shorter time to achieve the highest sensory level $(6.12 \pm 1.96 \mathrm{~min}$ vs $8.77 \pm 2.5 \mathrm{~min}, p=0.00)$, and a longer time till the first postoperative rescue analgesic need $(252.26 \pm 39.3 \mathrm{~min}$ versus $234.70 \pm 40.2 \mathrm{~min}, p=0.03)$. Group $P$ patients achieved statistically significantly higher level of sensory blockade and showed longer sensory block duration ( $216.30 \pm 30.8$ vs $199.44 \pm 23.8, p=0.003$ ). Adverse effects were comparable in both groups.

Conclusions: The sequential administration of fentanyl and hyperbaric bupivacaine improves the spinal block characters in patients subjected to lower limb orthopedic surgeries with comparable adverse effects profile in comparison to mixing both drugs.

Key-words: Bupivacaine, fentanyl, intrathecal, premixed, sequential, orthopedic surgeries.
\end{abstract}

improve the quality of spinal block including opioids. [2]

Coadministeration of intrathecal opioids and LAs have been found to produce a potent intra and postoperative analgesic synergism without further depression of efferent sympathetic activity, hence results in less adverse hemodynamic effects, even with sub therapeutic doses of LAs. [3] 
Fentanyl has been considered the intrathecal LAs adjuvant of choice owing to its potency, fast onset and short duration of action, and lower incidence of respiratory depression. $[4,5]$

Different factors have been shown to affect the intrathecal spread of LAs solution, e.g.; drug $\mathrm{pH}$, temperature, and baricity, patients' height and position after the injection, and the volume of injected solution. The action of the intrathecally administered solution may be affected by the routine practice of mixing the LAs with the adjuvant in the same syringe through altering its density and consequently its spread within the cerebrospinal fluid (CSF). [6, 7]

We hypothesized that the sequential administration of fentanyl and bupivacaine would improve the spinal block characters and early postoperative analgesia in comparison to mixing the two drugs.

The present trial aimed to compare the block characteristics, the postoperative analgesia duration, number of participants who requested for rescue analgesia within the $1^{\text {st }}$ six hours following surgery, and the side effects of sequential administration of intrathecal fentanyl and bupivacaine to the routine mixing of the two drugs in patients subjected to lower limb orthopedic surgeries under spinal anesthesia.

\section{Material and Methods}

After Institutional Review Board approval and getting informed written consent, American society of anesthesiologist (ASA) physical status 1 and 2 patients aged $20-65$ years, of either sex, who were subjected to elective lower limb orthopedic surgeries using spinal anesthesia, were recruited to this prospective, controlled, randomized, single blinded study. Patients with body mass index $\geq$ 30 , bleeding disorders, uncontrolled diabetes mellitus, peripheral neuropathy, known allergy to study drugs, presence of local infection, those on chronic opioid therapy, pregnant and drug abusers were excluded.
Participants were randomly divided into 2 groups ( $n=50$ each) using sequentially numbered opaque envelopes containing a randomization code to receive fentanyl and hyperbaric bupivacaine either as a premixed solution (Group $\mathrm{P}$ ) or in sequential manner (Group S) through separate syringe.

Preoperatively, patients were instructed on how to rate their pain using the visual analog scale (VAS), a $10 \mathrm{~cm}$ horizontal line where $0 \mathrm{~cm}=$ no pain and $10 \mathrm{~cm}=$ worst possible pain.

Participants' hemodynamics; heart rate (HR), noninvasive mean arterial blood pressure (MAP) and peripheral oxygen saturation were monitored. A venous access was secured in the arm and an infusion of lactated Ringer's solution as a coload $(10 \mathrm{ml} / \mathrm{Kg})$ was started.

Using complete aseptic technique and with the participants positioned sitting, spinal block was accomplished via a 25-gauge Quincke spinal needle at the L3-4 vertebral interspace via midline approach using $2.5 \mathrm{~mL}$ of $0.5 \%$ hyperbaric bupivacaine (HB) $(12.5 \mathrm{mg})$ plus fentanyl $25 \mu \mathrm{g}$ diluted in $0.5 \mathrm{ml}$ saline premixed in the same syringe in group $\mathrm{P}$ or $25 \mu \mathrm{g}$ fentanyl diluted in $0.5 \mathrm{ml}$ saline followed by injection of the same volume of bupivacaine through two separate syringes over $30 \mathrm{sec}$ in group S. Then all patients were positioned supine and surgery was allowed only after achieving at least T10 sensory blockade level.

Patients' hemodynamics, i.e. blood pressure, heart rate $(\mathrm{HR})$, and oxygen saturation, were monitored and registered every 2 minutes for the first 10 minutes and then every 5 minutes for the next 20 minutes then every $15 \mathrm{~min}$ till the end of the surgery. In case a clinically significant hypotensive episode (fall in mean arterial blood pressure (MAP) $>30 \%$ of baseline measure) was detected, a rapid infusion of $250 \mathrm{~m} \mathrm{~L}$ of Ringer lactate and/ or $5 \mathrm{mg}$ of intravenous ephedrine if hypotension persisted. Bradycardic (reduction of $\mathrm{HR}<50$ beats/min) was treated by intravenous atropine $0.02 \mathrm{mg} / \mathrm{kg}$. 
The spinal block progression was evaluated using loss to pinprick sensation every 2 min until the highest level had been fixed by successive tests. The time till achieving T10 sensory level in addition to the maximum block height were noted and recorded. The motor blockade was assessed using a modified Bromage scale $(0=$ no block, $1=$ partial block: patient unable to raise extended leg, II = almost complete: inability to flex knees, and III = complete block: inability to flex the ankle joint). Time to achieve Bromage III was recorded. The onset time of motor blockade and the motor blockade duration (the time from onset to complete recovery) were recorded.

Postoperatively, patients were observed for six hours. The times of two segments sensory regression from maximum level and motor block regression to Bromage 0 were noted and recorded .In case patients have reported pain VAS score $\geq 4$, a rescue analgesia of i.v. pethidine, $25 \mathrm{mg}$ was given. Time to the first rescue pethidine request and the number of participants who needed rescue analgesia were recorded.

The study primary outcome was the time to achieve T10 sensory block level. Secondary outcomes were other spinal block characteristics (block onset, maximum height and duration), number of participants that asked for rescue pethidine analgesia within the 1st 6 hours following surgery and the perioperative side effects e.g; hypotension, bradycardia, sheivering, nausea and/ or vomiting, sedation, and pruritus.

A blinded anesthetist not otherwise participating in patient care collected the study data.

Using Power Analysis and Sample Size software program (PASS) version 11.0.4 for windows (2011), the study sample size was calculated with the time to achieve T10 sensory block level as the primary outcome. Patients were allocated into two groups who received fentanyl and hyperbaric bupivacaine either as a premixed solution (Group P) or in sequential manner through separate syringe (Group S). The null hypothesis was considered as the absence of difference between both treatment modalities regarding the time to achieve sensory block at T10 level. An effect size of 0.6 (moderate effect size) as the target difference between both groups. A sample size of 45 patients / group was needed to achieve $80 \%$ power $(1-\beta$ or the probability of rejecting the null hypothesis when it is false) in the proposed study using two-sided twosample unequal-variance t-test with a significance level ( $\alpha$ or the probability of rejecting the null hypothesis when it is true) of $5 \%$. A $10 \%$ drop-out (5 patients) was expected in each group so 50 patients were enrolled into each group.

Data were statistically analyzed using SPSS version 20 software. A data normality test was done using the Kolmogorov-Smirnov test. Normally distributed continuous data were compared using the Independent- Sample T test and are expressed as mean (SD). Non-normally distributed data were analyzed using Mann-Whitney $U$ test and they are presented as median [interquartile range]. Nominal data were analyzed using the Chi-square test and are presented as number [percentage]. A statistical significance was considered at a $p$ value $<$ 0.05 .

\section{Results}

Fifteen patients have been excluded from the study (refused to participate or recent preoperative opioid use). One hundred patients fulfilling the inclusion criteria were allocated in a random way into one of the study groups. All patients completed the study with no recorded cases of failed block (Fig.1).

Both study groups were comparable in respect to the patients' demographics and surgical duration $(P>0.05)$ (Table1).

In comparison to group $\mathrm{P}$, group $\mathrm{S}$ patients had statistically significantly faster onset of both sensory $(4.58 \pm 1.5$ vs $5.40 \pm 1.8 \mathrm{~min}, \mathrm{p}=0.02)$ and motor block ( $5.79 \pm 1.5$ versus $6.64 \pm 1.9 \mathrm{~min}, \mathrm{p}$ $=0.01$ ), shorter time to achieve the highest sensory level $(6.12 \pm 1.96 \mathrm{~min}$ vs $8.77 \pm 2.5 \mathrm{~min}, \mathrm{p}=$ 
$0.00)$, and a longer time till the first postoperative rescue analgesic need $(252.26 \pm 39.3 \mathrm{~min}$ versus $234.70 \pm 40.2 \mathrm{~min}, p=0.03)$. However, group $P$ patients achieved statistically significantly higher sensory blockade level (T 6 ( T3 -T6) vs T 5 ( T4T7), $p=0.04$ ) and showed a longer sensory block duration $(216.30 \pm 30.8$ vs $199.44 \pm 23.8, p=$ 0.003) (Table 2).

There were no statistically significant differences between the two groups as regard time to two segment sensory regression, duration of motor block, and the number of participants that needed rescue analgesia within the first six hours after surgery (Table 2).

Perioperative adverse effects profile was comparable in both groups in terms of episodes of hypotension and bradycardia, shivering, nausea, vomiting, pruritus, and sedation (Table3).

Table1 Patients' characteristics and surgery duration

\begin{tabular}{|c|c|c|c|}
\hline & $\begin{array}{l}\text { Group P } \\
(\mathrm{n}=50)\end{array}$ & $\begin{array}{l}\text { Group S } \\
(\mathrm{n}=50)\end{array}$ & $P$ value \\
\hline Age (years) & $45.16 \pm 9.8$ & $48.20 \pm 8.3$ & 0.10 \\
\hline Sex; n (\%) & $29(58)$ & $34(68)$ & \\
\hline Male & $21(42)$ & $16(32)$ & 0.41 \\
\hline \multicolumn{4}{|l|}{ Female } \\
\hline Weight (kg) & $81.39 \pm 10.89$ & $77.76 \pm 8.43$ & 0.22 \\
\hline Height $(\mathrm{cm})$ & $166.77 \pm 5.12$ & $164.71 \pm 6.98$ & 0.27 \\
\hline $\operatorname{ASA}(\mathrm{I}, \mathrm{II})$ & $(34 / 16)$ & $(27 / 23)$ & 0.15 \\
\hline Surgery duration (min) & $84.23 \pm 10.24$ & $86.86 \pm 12.34$ & 0.45 \\
\hline
\end{tabular}


Table: 2 Spinal block characters and postoperative analgesia

\begin{tabular}{|c|c|c|c|}
\hline & $\begin{array}{l}\text { Group P } \\
(\mathrm{n}=50)\end{array}$ & $\begin{array}{l}\text { Group S } \\
(n=50)\end{array}$ & $P$ value \\
\hline Sensory block onset time ( $\min$ ) & $5.40 \pm 1.8$ & $4.58 \pm 1.5$ & $0.02^{*}$ \\
\hline Highest sensory level & $\mathrm{T}_{6}\left(\mathrm{~T}_{3}-\mathrm{T}_{6}\right)$ & $\mathrm{T}_{5}\left(\mathrm{~T}_{4}-\mathrm{T}_{7}\right)$ & $0.04^{*}$ \\
\hline Time till reaching highest sensory level (min) & $8.77 \pm 2.5$ & $6.12 \pm 1.9$ & $0.00^{*}$ \\
\hline Time to two segment sensory regression(min) & $133.50 \pm 29.7$ & $126.68 \pm 31.4$ & 0.26 \\
\hline Sensory block duration ( $\min$ ) & $216.30 \pm 30.8$ & $199.44 \pm 23.8$ & $0.003^{*}$ \\
\hline Motor block onset time ( $\min$ ) & $6.64 \pm 1.9$ & $5.79 \pm 1.5$ & $0.01^{*}$ \\
\hline Motor block duration ( min) & $131.40 \pm 27.6$ & $142.60 \pm 29.3$ & 0.06 \\
\hline Time to $1^{\text {st }}$ analgesic request ( $\mathrm{min}$ ) & $234.70 \pm 40.2$ & $252.26 \pm 39.3$ & $0.03^{*}$ \\
\hline No of patients requiring rescue analgesia in $1^{\text {st }} 6$ postoperative hours. & $11(22 \%)$ & $8(16 \%)$ & 0.44 \\
\hline
\end{tabular}

Data are mean $\pm \mathrm{SD}$, median (interquartile range) or number (\%). Group P: premixed group. Group S:

Sequential group. $\mathrm{P}<0.05$ means statistical significance 
Table 3 Perioperative adverse effects

\begin{tabular}{|c|c|c|c|}
\hline & $\begin{array}{l}\text { Group P } \\
(\mathrm{n}=50)\end{array}$ & $\begin{array}{l}\text { Group S } \\
(\mathrm{n}=50)\end{array}$ & $P$ value \\
\hline Intraoperative hypotension & $11(22 \%)$ & $8(16 \%)$ & 0.44 \\
\hline Intraoperative bradyardia & $4(8 \%)$ & $7(14 \%)$ & 0.33 \\
\hline Shivering & $8(16 \%)$ & $9(18 \%)$ & 0.79 \\
\hline Pruritus & $7(14 \%)$ & $11(22 \%)$ & 0.29 \\
\hline Nausea & $4(8 \%)$ & $3(6 \%)$ & 0.69 \\
\hline Vomiting & $1(2 \%)$ & 0 & 0.31 \\
\hline Sedation & 0 & 0 & NA \\
\hline
\end{tabular}

Data are number (\%). Group P: premixed group. Group S: sequential group.

$\mathrm{P} \geq 0.05$ means statistical insignificance. 


\section{Flow Diagram}

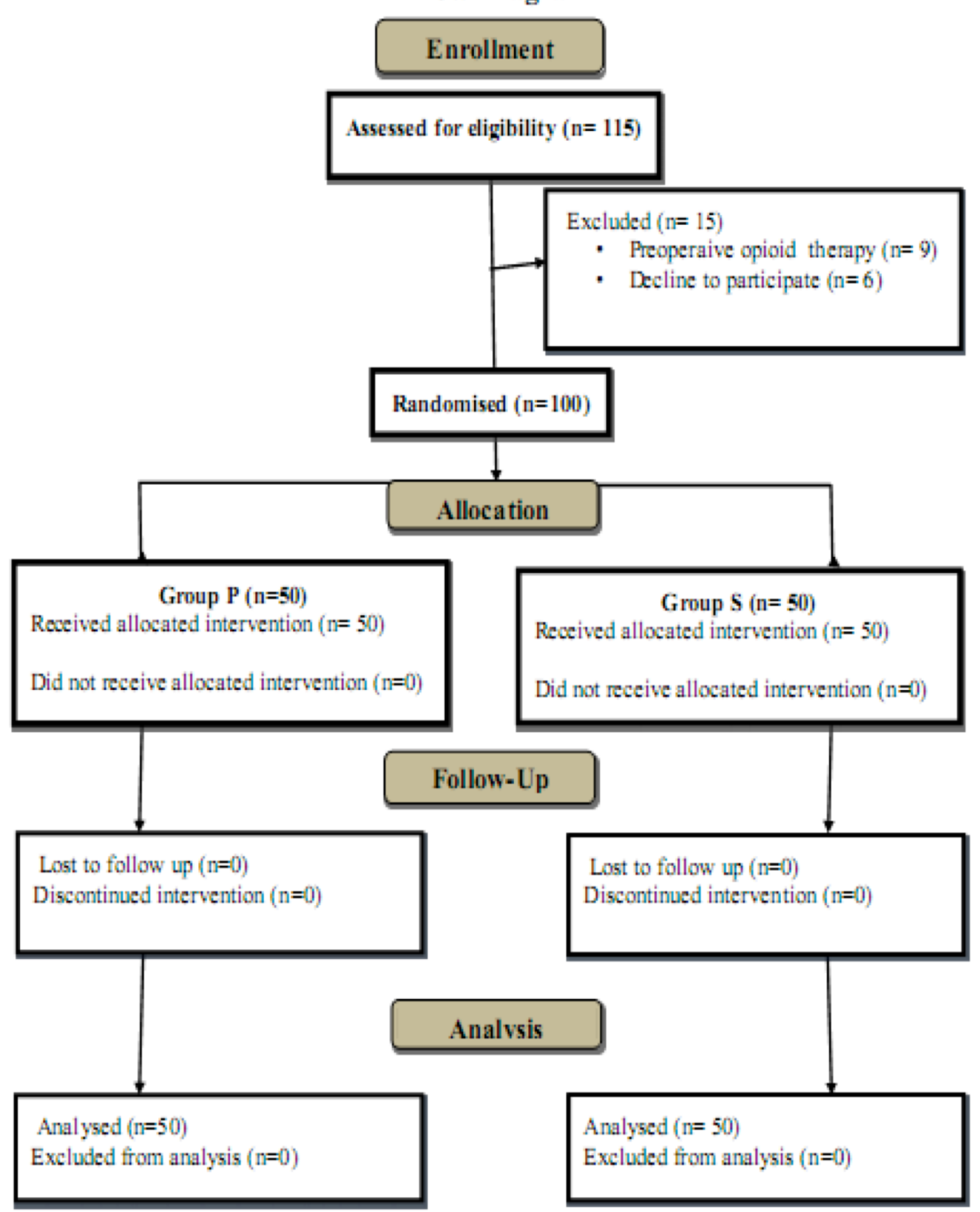




\section{Discussion}

The present prospective, controlled, randomized, single-blind trial has revealed that the sequential administration of fentanyl and bupivacaine intrathecally resulted in faster onset of both sensory and motor blocks, shorter time to achieve the highest sensory level, and a more prolonged time till the need for the first rescue analgesia with a comparable adverse effects profile.

Intrathecal fentanyl has been widely coadministered with hyperbaric bupivaciane during spinal anesthesia owing to its profound segmental antinociceptive action that enables using lower doses of LAs, enhances the intraoperative anesthesia quality, and extends the postoperative analgesia duration without further affection on the hemodynamics as it doesn't cause autonomic blockade. [8, 9]

In this study, the effects of altering the method of administration of intrathecal fentanyl and hyperbaric bupivacaine on the spinal block characteristics and the adverse effects profile were assessed.

The study results could demonstrate a faster onset to sensory and motor block with the sequential instillation of fentanyl and $\mathrm{HB}$ than with mixing them. This is in accordance with Upadye et al who have reached similar results. [10]

The demonstrated higher level of sensory block with the use of premixed solution in this study could be attributed to the reduction of bupivacaine density when mixed with opioid. Hypobaric bupivacaine has been found to achieve a higher block level in comparison to plain or hyperbaric bupivacaine when administered in the sitting position. [11] However the clinical significance of this observation in the current study could be questioned as the sensory level is higher by only one dermatome and no hemodynamic differences between both groups were observed.

The observed shorter time to achieve the highest sensory level with the use of sequentially adminis- terd drugs noted in this study could be explained by the preferential cephalad spread of fentanyl upon injecting it separately. This observation is supported by other investigators who had reported similar result when they had administered morphine and fentanyl separately during spinal anesthesia in parturients subjected to cesarean section than with the use of these opioids mixed with bupivacaine. [7]

The sequential administration of fentanyl and bupivacaine in this trial resulted in more prolonged duration of analgesia as the separate administration of fentanyl leads to greater spread and hence formation of firmer opioid receptor bonds giving denser and more prolonged block in contrast to the less profound block induced by the diluted mixture of fentanyl and HB. Similarly, Gray et al have revealed increased duration of postthoracotomy analgesia with the intrathecal use of hypobaric morphine (in normal saline) in comparison to the use of hyperbaric one (combined with dextrose) due to change of its distribution upon being mixed with dextrose.[12] Chaudhry et al., and Sachan et al., have demonstrated more prolonged analgesia duration with the sequential use of dexmedetomidine and clonidine during spinal anesthesia for lower limb orthopedic surgeries and cesarean section respectively, compared to their mixed use with hyperbaric bupivacaine. [13, 14]

\section{Conclusion:}

The sequential administration of fentanyl and hyperbaric bupivacaine improves the spinal block characters in patients subjected to lower limb orthopedic surgeries with comparable adverse effects profile in comparison to mixing both drugs.

\section{References}

1. Grande RP, Wig J, Yaddanapudi LN. Evaluation of bupivacaine- clonidine combination for unilateral spinal anesthesia in lower limb orthopedic surgery. J Anaesth Clin Pharmacol 2008; 24:155-8. 
2. Sapate M, Sahu P, Shah B, Suryawanshi C, Kulkarni A, Panditrao MM. Evaluation of bupivacaine-clonidine combination for unilateral spinal anesthesia in lower limb below knee orthopedic surgery. Saudi J Anaesth 2014; 8:384-7.

3. Singh H1, Yang J, Thornton K, Giesecke AH, Intrathecal fentanyl prolongs sensory bupivacaine spinal block. CanJ Anaesth 1995, 42 (11):987-91.

4. Benhamou D, Thorin D, Brichant JF, Dailland $\mathrm{P}$, Milon $\mathrm{D}$, Schneider M. Intrathecal clonidine and fentanyl with hyperbaric bupivacaine improves analgesia during cesarean section. Anesth Analg 1998; 87:609-13.

5. Selvaraju KN, Sharma SV. Comparison of forced expiratory spirometric flow changes following intrathecal bupivacaine and bupivacaine with fentanyl. South Afr J Anesth Analg 2008; 14:33-7.

6. Greene NM. Distribution of local anesthetic solution within the subarchnoid space. Anesth Analg 1985; 64:715-30.

7. Desai $\mathrm{S}$, Lim $\mathrm{Y}, \mathrm{Tan} \mathrm{CH}$, Sia AT. A randomized controlled trial of hyperbaric bupivacaine with opioids, injected as either a mixtyre or sequentially, for spinal Anesthesia for caeserian section. Anaesth Intensive Care 2010; 38:2804.

8. Hunt CO, Naulty JS, Bader AM. Perioperative analgesia with subarachnoid fentanyl with bupivacaine for Caesarian delivery. Anesthesiolo- gy $1989 ; 71 ; 535-540$.

9. Morgan M. The rational use of intrathecal and extradural opioids. $\mathrm{Br} J$ Anaesth 1989; 63: 165-188.

10. Upadya M, NeetaS, Manissery JJ, Kuriakose N, Singh RR. A randomized controlled study comparing intrathecal hyperbaric bupivacainefentanyl mixture and isobaric bupivacainefentanyl mixture in common urological procedures. Indian J Anaesth. 2016; 60:44-9.

11. Hallworth SP, Fernando $R$, Columb $M$, Stocks GM. The effect of posture and baricity on the spread of intrathecal bupivacaine for elective cesarean delivery. Anesth Analg 2005; 100:1159-1165.

12. Gray JR, Fromme GA, Nauss LA, Wang JK, IIstrup DM. Intrathecal morphine for postthoracotomy pain. Anesth Analg 1986; 65:873876.

13. Gunjan Chaudhry, Deepak Singla, Jagdish Dureja, Pranav Bansal \& Kirti Ahuja et al., Efficacy of premixed versus sequential administration of dexmedetomidine as an adjuvant to intrathecal hyperbaric bupivacaine in lower limb surgery, Southern African Journal of Anesthesia and Analgesia 2016;22(3):81-85.

14. Sharma SK, Sehera $H$, Sharma $M$, et al.,Comparison of premixed and sequentially intrathecal administration of clonidine with hyperbaric Bupivacaine in caesarean sections, International Multispecialty Journal of Health $2015 ; 1: 3: 20-24$. 